\title{
Optimal Customer Satisfaction in Rural Travelling Decision-Making based on Logistic Regression Model
}

\author{
Wei Liu* \\ Management Engineering Department, Henan Mechanical and Electrical Engineering College, Xinxiang, 453003, \\ China
}

\begin{abstract}
With the economic development and the improvement of residents' income level, China's tourism industry is now entering into a new historical period. In this paper, we construct an empirical analysis model about urban residents' rural travelling decision-making, and then analyze influential factors mechanism by using logistic regression model. Based on the analysis of rural travelling decision-making behaviors, the result shows that the variables as disposable income, discretionary time of urban residents, infrastructure, traffic condition, tourism culture and tourism environment are significantly affecting rural travel decision-making, which is at $10 \%$ significant level; meanwhile, other variables as environmental quality, policy and media publicity is not obvious.
\end{abstract}

Keywords: Customer satisfaction, logistic regression model, rural traveling, travel decision-making.

\section{INTRODUCTION}

With the economic development and the improvement of residents' income level, China's tourism industry will enter into a new historical period. As the special product of tourism, rural tourism not only has an industrial function to help rising farmers' income and promote employment, but also has the leisure function to let urban residents go back to the nature and feel relax. As the demand side of rural tourism, urban residents' travelling decision-making has important theoretical and practical value to the development of rural tourism. In view of this, we adopt the theoretical and empirical research, using questionnaires and empirical methods to explore the influential factors in decision-making process of urban residents' rural travelling, based on the samples of Henan province, China. Based on this research, we put forward some relevant policy suggestions about the development of rural tourism and increasing the rural travelling consumption.

Theoretical study of rural tourism is really mature and perfect; the research method is gradually in-depth and detailed. In theoretical aspect, some scholars started their research from the areas as sustainable development of rural tourism; such as the integration of rural tourism (IRT). IRT refers to the rural tourism stakeholders in the integration of rural area natural, economic, social and cultural resources and utilized these resources [1], it emphasis the profits but also enable them to obtain the effective protection, so it is a kind of sustainable tourism development model. Saxena (2008) make the case study of rural tourism by using England and the Welsh border area as an example, and points out that the integrated rural tourism network system is important [2].

*Address correspondence to this author at the Management Engineering Department, Henan Mechanical and Electrical Engineering College, Xinxiang, 453003, China; Tel: +86-03733392102; Fax: +86-03733392102; E-mail: liuweihenan33@163.com
Farm tourism as a subset of the rural tourism, it is the embodiment of the typical characteristics of township enterprises: such as the small scale local roots and affiliated local tradition; but it is as natural and social tourism representative, rural tourism also has the different characteristics [3]. There are also some scholars introduce the multi criteria modeling methods of the service quality evaluation, and try to improve the quality of service [4]. However, we should also pay attention that there exist different kinds of farm families, so we should realize tourism diversification. On this basis, agriculture and tourism industries should support each other, and realize rural tourism common development [5].

The relationship between rural tourism and agricultural tourism mainly divided into the following five types, it mainly depend on whether participate in farm work, such as: nonfarm tourism, agricultural tourism, indirect farming tourism, tourism with farm work, real farm work and agricultural tourism [6]. Cultural rural tourism is also a unique part in rural tourism, it not only for entertainment, but pays more attention to cultural communication [7]; some scholars pointed out that unique cultural resources and community cooperation is very effective in cultural rural tourism, and make the case study from Canada [8]. Actually, rural tourism has become an important factor to promote economic growth in rural areas and remote areas, and is also conducive to the culture development in these regions, unique cultural resources and community cooperation is very successful for rural tourism [9]. There are also many scholars use empirical analysis, and focused on tourism stakeholders and the tourists as the study object, they mainly analyzes the consumer behavior of tourists [10], and make customer segmentation. Also, there are many scholars who research on the tourist motivation [11, 12], and analyze the preference of different tourist, also there are some scholars research the related factors that affect satisfaction in rural tourism [13]. 
In terms of rural tourism, there exists an important theory as tourism decision theory. Decision analysis pointed out that the people's behavior and decision is based on the analysis of collected information, people make decisions by evaluating each possible outcome of the advantages and disadvantages, they finally selected the best from a series of choices, which is to achieve their goals as the most suitable solution. The decision behavior is called optimal subjective expected utility choice [14-15]. But this theory is not considered the decisive constraints, and has limitations to a certain extent. Regret Theory is a new theory to solve this constraint; it can solve these constraints by adjusting the decision of risk or uncertainty. There are also many scholars research about the tourism decision model, Wahab and Crampon (1976) put forward the tourists decision is just as a rational economic person tries to solve problems and advance the plan to reduce the risk, this behavior is in order to make their purchase behavior to achieve the maximized utility. The tourism decision model is recognized as the bounded rationality in academic community in the earliest [16]. From a more practical point of view, bounded rationality is because of the time limitation, cognitive ability limited and incomplete information, so that personal decisions is to make their own satisfaction instead of the optimal one. Related to this point of view, Simon (1979) pointed out that the rational tourism by tourists including many conditions constraints such as stimulation, social psychological factors and the environment restriction, and first pay attention to limiting factor on travel decision [17]. Mayo and Javis (1981) make empirical research about tourism decision-making, and find out that under the limited rational decision, tourism decisionmaking is a common result of social factors and individual psychological factors [18]. Moutinho (1987) discussed the social impact on travel decision-making [19], and proved that the social influence is the important factors in the longterm decision-making activities. Then, Paul (1997) construct a comprehensive model including internal factors, cognitive structure and the external factors, and try to find out the factors which influence decision-making process, they also put forward some suggestions about how to improve the tourist satisfaction [20].

\section{QUESTIONAIRE AND MODEL DESIGE}

Questionnaire design: According to previous research on the questionnaire survey, the design of this questionnaire is divided into five steps: (1) design several categories according to the existing literature and research; (2) integrate literature and refine each category; (3) assume the logical relationship existing between variables, then set up related issues; (4) using expert interviews, adoption their opinions and suggestions, then improve the questionnaire; (5) determine the measurement problem for each variable by exploratory factor analysis, and determine the final questionnaire. The idea of questionnaire design come from arranging and analyzing previous literature, it refers to customer satisfaction in rural travel decisions. We obtains the preliminary questionnaire by summarizing and comparing existing results, then use expert interviews to increase the appropriateness of the questionnaire items.

In order to improve the questionnaire as simple and effective, we will release a part of the pretest questionnaire, through the screening of the results to determine the final items. Finally, we will release a lot of formal questionnaire and collect data, then make reliability test, validity test, correlation analysis and regression analysis by using SPSS software.

The main variables and symbolic hypothesis: on the basis of comprehensive analysis on the influencing factors, the mechanism of urban residents' rural-travel decision can be expressed as:

$$
Y=F\left(X_{A}, X_{B}, X_{C}, X_{D}, Z\right)
$$

In this formula, $\mathrm{Y}$ represents the result of the urban rural travel decisions; Xa represents resident characteristic variables; $\mathrm{Xb}$ represents rural tourism destination; $\mathrm{Xc}$ represents rural tourism channel;Xd represents rural tourism environment; $Z$ represents dummy variables as regional location. Let $\mathrm{Y}$ denote the city residents village traveling decision making results ( $\mathrm{Y}=1$ means travel, $\mathrm{Y}=0$ means not travel), $\mathrm{Xi}$ represents the factors $(i=1,2, \ldots, I)$, then the formula can be further expressed as:

$$
Y=\sum \beta_{i} x_{i}+\alpha
$$

Among them, the beta I represents $\mathrm{Xi}$ changes on rural urban residents travel the influence degree of probability; Alpha as independent random errors, meet with mean zero, variance of 1 standard normal distribution, it represents some of the potential not observed variables and data error; Formula (2) is an empirical analysis about application of econometric model. Based on analysis, this paper examine variables to make the following choice, refer to the related research and the coefficient of symbols make the assumption, as shown in Table $\mathbf{1}$.

Further, for this kind of phenomenon of binary discrete quantity analysis, this study adopts the binary Logistic model to analyze urban residents travel decision-making, in order to more objectively analyze the effect of various influence factors of residents' travel decision direction, a more accurate measure its impact. Remember the conditional probability of the incident $\mathrm{P}(\mathrm{y}=1 \mid \mathrm{xi})=\mathrm{Pi}$, can get the following logistic return model:

$$
\begin{gathered}
p_{i}=\frac{1}{-\left(\alpha+\sum_{i=1}^{m} \beta_{i} x_{i}\right)}=\frac{e^{\alpha+\sum_{i=1}^{m} \beta_{i} x_{i}}}{1+e^{\alpha+\sum_{i=1}^{m} \beta_{i} x_{i}}} \\
1-p_{i}=1-\frac{e^{\alpha+\sum_{i=1}^{m} \beta_{i} x_{i}}}{1+e^{\alpha+\sum_{i=1}^{m} \beta_{i} x_{i}}}=\frac{1}{1+e^{\alpha+\sum_{i=1}^{m} \beta_{i} x_{i}}}
\end{gathered}
$$

In this formula, $\mathrm{Pi}$ representatives the probability of events as observation $\mathrm{i}, 1$ - Pi represent in the probability of events does not occur, they are all made of the independent variable xi nonlinear function. The ratio of the incidence of the incident and $\mathrm{Pi} /(1-\mathrm{Pi})$ referred to as the occurrence of the event, for the Odds. Odds have no upper bound and positively $(0<\mathrm{Pi}<1)$, the logarithmic transformation of logistic linear model can be shown as: 
Table 1. The main variables and symbolic hypothesis.

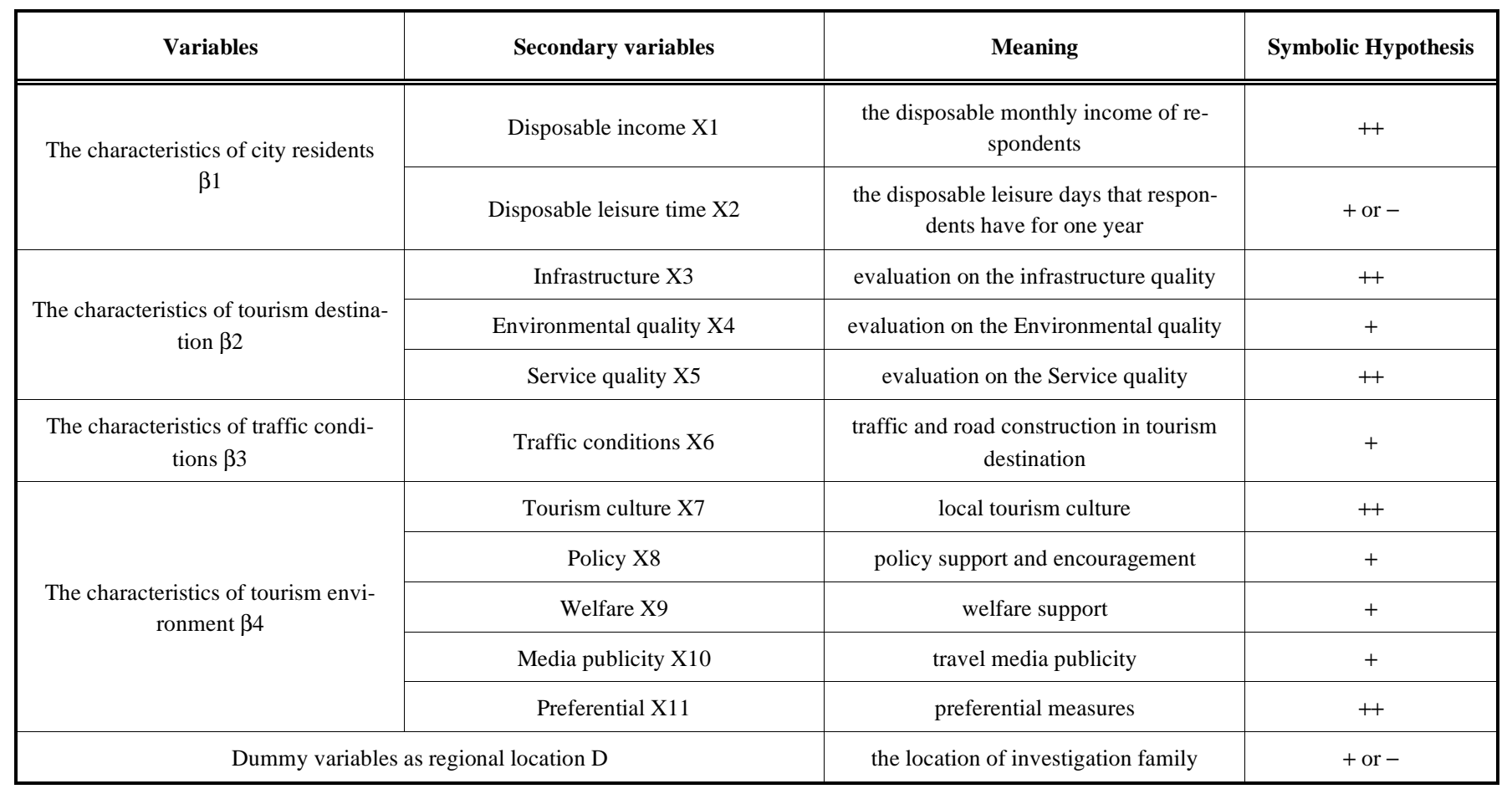

Note: “++" means the strong positive influence; "+" means positive influence; "-" means negative influence

$\operatorname{Ln}\left(\frac{p_{i}}{1-\mathrm{p}_{\mathrm{i}}}\right)=1-\frac{e^{\alpha+\sum_{i=1}^{m} \beta_{i} x_{i}}}{1+e^{\alpha+\sum_{i=1}^{m} \beta_{i} x_{i}}}=\frac{1}{1+e^{\alpha+\sum_{i=1}^{m} \beta_{i} x_{i}}}$

\section{RESULTS AND DISCUSSION}

In order to analyze the influencing factors of urban rural travel decision-making, in this section, we first analyze related factors affecting urban residents travel decision-making and construct the econometric model, and then we make empirical analysis on the basis of theoretical model. Through empirical research, we discuss the influence factors of urban rural travel decision-making mechanism. The content of this section is based on the on-the-spot investigation data analysis.

Data sources and sample: This paper taking 2014 (1-9 months) as the survey time period, and the questionnaire was collected when urban residents participated in the rural tourism. The author choose Xinxiang city of Henan province as the main sampling area, we mainly choose locations as concentrated large supermarkets, shopping malls, and rural tourism attractions. 200 questionnaires were distributed by field questionnaire survey, recovery of 167 copies, no answer or reply invalid questionnaire is 28 , effective questionnaire 139 , effective questionnaire rate was $69.5 \%$.

Statistical analysis of urban residents' basic characteristic: From the result of the survey, the age trend of urban residents participate in the rural tourism was younger (average age 32.62 years), with higher disposable monthly income (2104.76 RMB), disposable leisure time is more abundant (79.04), as shown in Table 2. From Table 3, We can get the occupation distribution percentage of survey, as civil servants $(12.23 \%)$, Enterprise staff $(24.46 \%)$, Business \& service personnel $(40.28 \%)$ and students $(14.38 \%)$, it will illustrate that the occupational structure will influence income and leisure time directly, then influence travel behavior of tourists. Then we analyze the education background of the questionnaire participants, The result shows the proportion of people that achieved higher education is $82 \%$, among them, college education accounted for $48.92 \%$, graduate education accounted for $33.09 \%$. And the proportion of people who has high school diploma is only $17.98 \%$, which fully shows that the education background will also influence the rural tourism behavior, and there exist a positive correlation between the education background and travel behavior. As shown in Table 3 .

Describe the statistic characteristic of rural tourism destinations: This paper selects the variables as infrastructure, rural tourism destination of the ecological environment, quality of service as to study the factors affecting the destination variable. From the statistical analysis in Table 4, we can get that the variable as "environment quality" get the highest mean score as 83.46 , it shows that environmental quality is the most import at factor in rural tourism decision, so the ecological environment should meet consumer's pursuit of natural experience. Secondly, the quality of service is also an important factor, and the mean score is 71.03. It shows that residents will also pay attention to the local service quality when they make decision, so service quality is closely related to the travel decision. Finally, the infrastructure quality gets the lowest average as 67.82, it show that local rural tourism also need more relevant public facilities. The result was shown in Table 4. 
Table 2. Statistical analysis of urban residents' basic characteristic.

\begin{tabular}{|c|c|c|c|c|}
\hline Personal Characteristics & Minimum & Maximum & Average & S.D. \\
\hline \hline Age & 17 & 59 & 32.62 & 8.235 \\
\hline Disposable monthly income & 0 & 20000 & 2104.76 & 3162.579 \\
\hline Discretionary time & 50 & 150 & 79.04 & 67.821 \\
\hline
\end{tabular}

Table 3. Occupation and education background analysis of Urban residents.

\begin{tabular}{|c|c|c|c|c|c|}
\hline \multirow{4}{*}{ Occupation } & civil servants & 17 & 12.23 & 12.23 & 12.23 \\
\hline & Enterprise staff & 34 & 24.46 & 24.46 & 36.69 \\
\hline & Business \& service personnel & 56 & 40.28 & 40.28 & 76.97 \\
\hline & others & 12 & 8.65 & 8.65 & 100 \\
\hline \multirow{3}{*}{ Education } & High School & 25 & 17.98 & 17.98 & 17.98 \\
\hline & Undergraduate & 68 & 48.92 & 48.92 & 66.91 \\
\hline & Graduate & 46 & 33.09 & 33.09 & 100 \\
\hline
\end{tabular}

Table 4. Describe the statistic characteristic of rural tourism destinations.

\begin{tabular}{|c|c|c|c|c|}
\hline \multirow{2}{*}{} & Infrastructure & Environmental Quality & Service Quality \\
\hline \hline \multirow{3}{*}{ Samples } & Effective number & 139 & 136 & 139 \\
\cline { 2 - 5 } & Missing number & 0 & 3 & 0 \\
\hline Mean & 67.82 & 83.46 & 71.03 \\
\hline \multicolumn{2}{|c|}{ Standard deviation } & 14.57 & 8.42 & 15.13 \\
\hline \multicolumn{2}{c|}{ Minimum } & 10 & 50 & 20 \\
\hline \multicolumn{2}{c|}{ Maximum } & 100 & 100 & 100 \\
\hline
\end{tabular}

Table 5. Describe of the tourism environment supporting factors.

\begin{tabular}{|c|c|c|c|c|}
\hline & & Number & Response Percentage & Case Percentage \\
\hline \hline \multirow{3}{*}{\begin{tabular}{c} 
The characteristics of tour- $\begin{array}{c}\text { ism environment } \\
\text { ismy }\end{array}$ \\
\cline { 2 - 5 }
\end{tabular}} & Tourism culture & 68 & $39.53 \%$ & $71.08 \%$ \\
\cline { 2 - 5 } & Policy & 7 & $4.06 \%$ & $9.12 \%$ \\
\cline { 2 - 5 } & Media publicity & 32 & $28.48 \%$ & $34.65 \%$ \\
\hline
\end{tabular}


Table 6. The model of goodness of fit evaluation.

\begin{tabular}{|c|c|c|c|c|c|c|}
\hline & \multicolumn{3}{|c|}{ Hosmer and Lemeshow Test } & \multicolumn{3}{c|}{ Model Summary } \\
\hline \hline Step & Chi-square & df & Sig. & -2Loglikelihood & Cox\&Snell & R2 Nagelker ke R2 \\
\hline 1 & 5.723 & 6 & .721 & 61.687 & .514 & .695 \\
\hline
\end{tabular}

Table 7. The results of regression model.

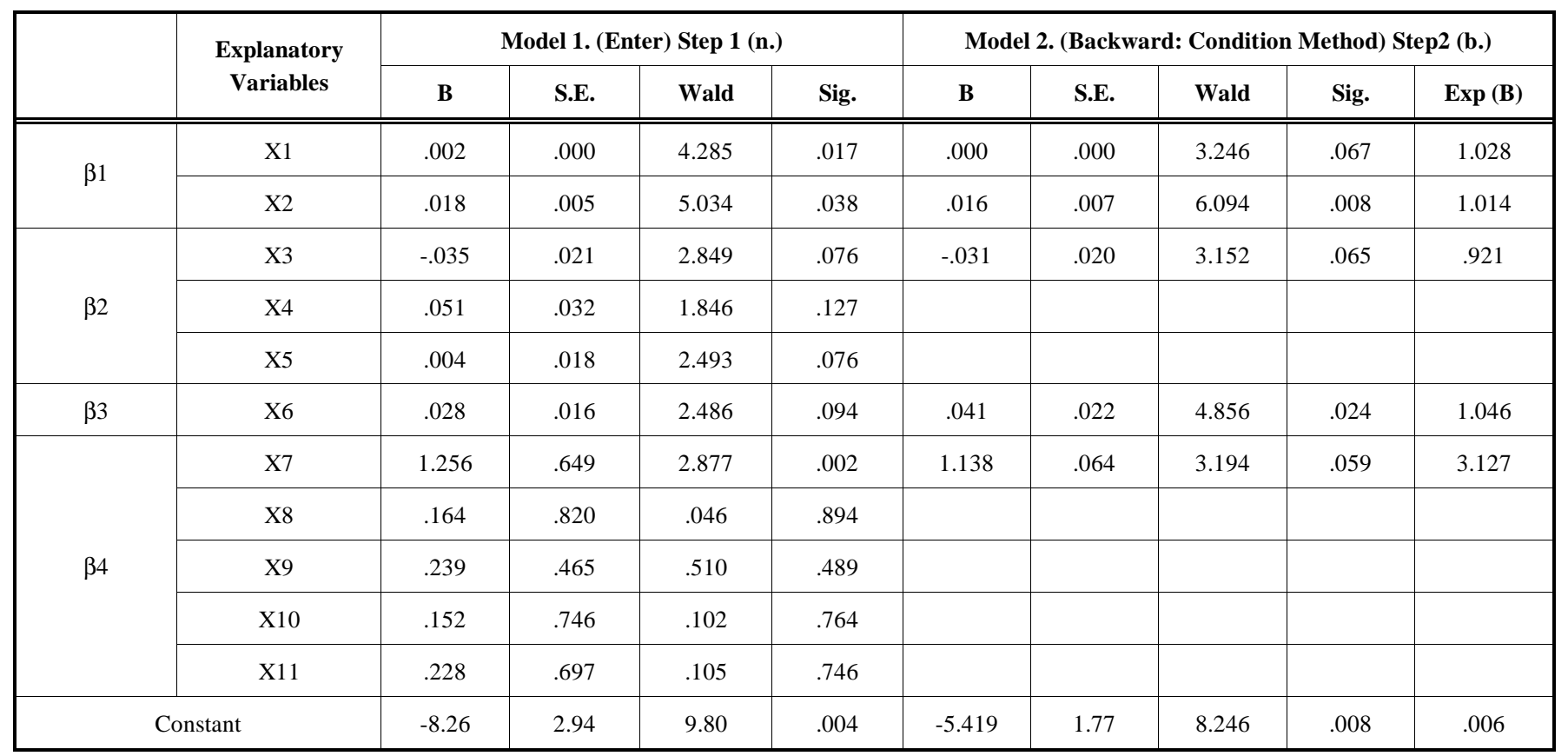

Describe of the tourism environment supporting factors: then we analyze the frequency of the tourism environment supporting factors, in support of the influence factors of rural tourism travel decision environment, $71.08 \%$ of people choose the "local tourism and cultural atmosphere" option, and $39.53 \%$ of people choose the total environment factors. Therefore, in the process of rural travel decision behavior, the local tourism culture the atmosphere is the main factor of environment support. The third factor is the "tourism media propaganda" option (response percentage is $28.48 \%$ ), also the influencing factors showed that the media is an important factor. Urban residents consider whether or not to travel mainly from their own point of view, and the implementation of the national macro policies for their travel decision behavior is not obvious, so "the state and government policy support" is the lowest frequency (response rate of $4.06 \%$ ). The result was shown in Table 5.

The goodness of fit analysis in the model: According to the output analysis, fitting statistics (Hosmer and Lemeshow Test) values $(\rho=0.721)>0.05$, unable to reject the null hypothesis, shows the probability to obtain the expected frequency and there was no statistically significant difference between the observed frequency, namely survey data model fitting is good, as shown in Table 6. The final test of goodness of the model is 61.687, Model of the fit of the data is more ideal; the following reference Nagelkerke R2 statistic a value as 0.695 , data also shows that a better fitting effect.

The results of regression model: Urban residents have willingness to travel under the premise of urban rural travel decision-making a result (travel or not travel) is essentially a dichotomous variable. The dependent variable value range of the traditional regression model between is infinite and negative infinity, in this apparently doesn't fit, so this paper USES the binary Logistic regression analysis model is analyzed, and using the maximum likelihood estimation method is used to estimate the parameters. This study uses SPSS17.0 statistical software for survey data processing, first choose forced into law, will have to examine variables one-time into the Logistic regression model analysis, it is concluded that model 1; After the second choice to gradually selection method, will examine all variables in the regression model analysis, investigation variables significantly model 2 , as shown in Table 7.

Overall, the regression model of the empirical results with the above assumptions, under the $10 \%$ significant level, the disposable income, discretionary time, infrastructure condition, tourism traffic, tourism culture is effective variables to decision-making, and will have significantly influence to rural tourism decision-making, the effects of other variables is not obvious. 


\section{CONCLUSION}

Based on the theoretical analysis, this paper sets an empirical analysis model about urban residents' rural travelling decision-making, and analyzes influential factors mechanism. According to designing questionnaires; we collect urban residents' data and use statistical analysis software SPSS17.0 for data analysis. Then plug all of the variables measured on examination into Logistic regression model, it is found that the model regression results are basically consistent with the hypothesis of all variables, the result shows that personal characteristics of urban residents, discretionary leisure time, traffic, tourist conditions are significant variables which affect the rural travelling decision-making behavior.

From the empirical analysis, the result shows that: 1 . The regression coefficient of urban residents personal disposable income is positive and the sig value is 0.067 , it means that the premise of the ceteris paribus, disposable income of urban residents in the higher possibility of the participation of rural tourism is more big, and consistent with the above hypothesis.2. Discretionary time is the most significant urban residents' personal characteristics vector variables, the sig value of 0.008 , with significant statistical significance; and the regression coefficient is positive, it shows that under the condition of other factors constant, the more discretionary time, city residents choose the higher the probability of rural tourism. 3. Infrastructure condition variables sig value is less than 0.1 , the regression coefficient value is -0.035 , the symbol for the negative with the above assumptions, investigate its reason, probably because the city residents to participate in the study area of rural tourism is more focus on rural tourism ecological environment quality, and less attention on the infrastructure, or because the region's infrastructure is relatively perfect rural tourism attractions, so this variable to greatly reduced the impact of urban rural travel decisions.4. from the model results, the environment quality of the rural tourism destination regression coefficient and maximum value symbol as 0.051 , it means that in the case of other conditions unchanged, the environment quality of the rural tourism destination will effect on the decision-making in rural tourism. The regression coefficient of rural personnel's service quality is positive; its connotation is that when other conditions unchanged, rural talent has played a positive role in improving the service quality of rural travel.

\section{CONFLICT OF INTEREST}

The authors confirm that this article content has no conflict of interest.

\section{ACKNOWLEDGEMENTS}

Declared none.

\section{REFERENCES}

[1] M. Cawley, and D. A. Gillmor, "Integrated rural tourism: Concepts and practice", Annals of Tourism Research, vol. 35, pp. 316-337, 2008.

[2] G. Saxena, and B. Ilbery, "Integrated rural tourism: a border case study", Annals of Tourism Research, vol. 35, pp. 233-254, 2008.

[3] P. A. Nilsson, "Staying on farms: An ideological background", Annals of Tourism Research, vol. 29, pp. 7-24, 2002.

[4] C. Rozman, "A multi-criteria assessment of tourist farm service quality”, Tourism Management, vol. 30, pp. 629-637, 2009.

[5] R. Sharpley, and D. Jepson, "RURAL TOURISM: A spiritual experience?" Annals of Tourism Research, vol.37, pp.10-16, 2010.

[6] D. Kahneman, and A. Tversky, "Prospect theory: An analysis of decisions under risk", Econometrical, vol. 47, pp. 262-291, 1979.

[7] C. Hunter, and K. Blackstock, "A typology for defining agritourism”, Tourism Management, vol. 31, pp. 754-758, 2010.

[8] D. Getz, and J. Carlsen, "Characteristics and goals of family and owner-operated businesses in the rural tourism and hospitality sectors", Tourism Management, vol. 21, pp. 547-560, 2010.

[9] R. MacDonald, and L. Jolliffe, "Cultural rural tousrism: evidence from Canada", Annals of Tourism Research, vol. 30, pp. 307-322, 2003.

[10] F. Isabelle, "A benefit segmentation of tourists in rural areas: a Scottish perspective", Tourism Management, vol. 26, pp. 335-346, 2005.

[11] L. Molera, and I. P. Albaladejo, "Profiling segments of tourists in rural areas of South-Eastern Spain", Tourism Management, vol. 28, pp. 757-767, 2007.

[12] D. B. Park, and Y. S. Yoon, "Segmentation by motivation in rural tourism: A Korean case study", Tourism Management, vol. 30, pp. 99-108, 2009.

[13] I. P. Albaladejo-Pina, "Tourist preferences for rural house stays: Evidence from discrete choice modelling in Spain", Tourism Management, vol. 30, pp. 805-811, 2009.

[14] D. Maria, L. Marta, and P. Andres, "The role of motivation in visitor satisfaction: Empirical evidence in rural tourism", Tourism Management, vol. 31, pp. 547-552, 2010.

[15] W. Edwards, "The theory of decision-making. Psychological Bulletin”, Tourism Management vol.51, pp. 380-417, 1954.

[16] S. Wahab, and L. J. Crampon, Tourism marketing. London: Tourism International Press, 1976.

[17] H. A. Simon, Models of man: Social and rational, New York: Wiley, 1957

[18] E. J. Mayo, and L. P. Jarvis, "The psychology of leisure travel", Boston, MA: CBI Publishing Company Inc, 1981.

[19] L. Moutinho, "Consumer behavior in tourism", European Journal of Marketing, vol. 21, pp. 3-44, 1987.

[20] R. T. Paul, G. Shaw, and A. M. Williams, "Tourist group holiday decision-making and behavior: the influence of children", Tourism Management, vol. 18, pp. 287-297, 1997.

(C) Wei Liu; Licensee Bentham Open.

This is an open access article licensed under the terms of the Creative Commons Attribution Non-Commercial License (http://creativecommons.org/licenses/by-nc/3.0/) which permits unrestricted, non-commercial use, distribution and reproduction in any medium, provided the work is properly cited. 